\title{
Using polygenic scores for identifying individuals at increased risk of substance use disorders in clinical and population samples
}

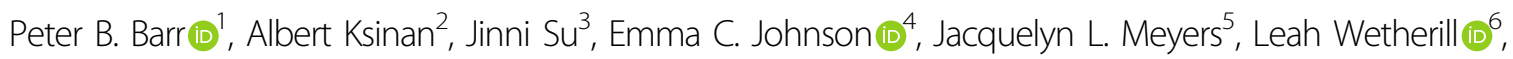
Antti Latvala (10 ${ }^{7,8}$, Fazil Aliev (1) ${ }^{1,9}$, Grace Chan (10 ${ }^{10}$, Samuel Kuperman ${ }^{11}$, John Nurnberger ${ }^{6,12,13}$, Chella Kamarajan (10) Andrey Anokhin ${ }^{4}$, Arpana Agrawal $\mathbb{1}^{4}$, Richard J. Rose ${ }^{14}$, Howard J. Edenberg $\mathbb{1}^{6,15}$, Marc Schuckit ${ }^{16}$, Jaakko Kaprio ${ }^{7,17}$ and Danielle M. Dick ${ }^{1,18}$

\begin{abstract}
Genome-wide, polygenic risk scores (PRS) have emerged as a useful way to characterize genetic liability. There is growing evidence that PRS may prove useful for early identification of those at increased risk for certain diseases. The current potential of PRS for alcohol use disorders (AUD) remains an open question. Using data from both a populationbased sample [the FinnTwin12 (FT12) study] and a high-risk sample [the Collaborative Study on the Genetics of Alcoholism (COGA)], we examined the association between PRSs derived from genome-wide association studies (GWASs) of (1) alcohol dependence/alcohol problems, (2) alcohol consumption, and (3) risky behaviors with AUD and other substance use disorder (SUD) criteria. These PRSs explain $~ 2.5-3.5 \%$ of the variance in AUD (across FT12 and COGA) when all PRSs are included in the same model. Calculations of area under the curve (AUC) show PRS provide only a slight improvement over a model with age, sex, and ancestral principal components as covariates. While individuals in the top 20,10, and 5\% of the PRS distribution had greater odds of having an AUD compared to the lower end of the continuum in both COGA and FT12, the point estimates at each threshold were statistically indistinguishable. Those in the top 5\% reported greater levels of licit (alcohol and nicotine) and illicit (cannabis and opioid) SUD criteria. PRSs are associated with risk for SUD in independent samples. However, usefulness for identifying those at increased risk in their current form is modest, at best. Improvement in predictive ability will likely be dependent on increasing the size of well-phenotyped discovery samples.
\end{abstract}

\section{Introduction}

Alcohol misuse is one of the leading contributors to preventable mortality and morbidity worldwide ${ }^{1-3}$. Identifying individuals at heightened risk for developing alcohol-related problems remains an important goal of medical practitioners. One important risk factor for alcohol misuse is one's own genetic liability. Twin and

Correspondence: Peter B. Barr (pbarr2@vcu.edu)

'Department of Psychology, Virginia Commonwealth University, Richmond, VA, USA

${ }^{2}$ Department of Health Behavior and Policy, Virginia Commonwealth University, Richmond, VA, USA

Full list of author information is available at the end of the article family studies indicate that genetic influences on alcohol use disorders (AUD) account for $\sim 50 \%$ of the variation in the population ${ }^{4}$. Genome-wide association studies (GWASs) have identified multiple variants associated with AUD $^{5-7}$, alcohol consumption ${ }^{7,8}$, and maximum alcohol intake 9 . Using information from these GWASs, we are now able to aggregate risk across the genome by creating polygenic risk scores (PRS) in independent samples ${ }^{5,6,8,10}$.

Beyond being useful for research purposes, researchers have begun to examine the potential of PRS to predict risk for medical outcomes in clinical settings. PRS for coronary artery disease (CAD), atrial fibrillation (AF), type 2 diabetes (T2D), inflammatory bowel disease (IBS), and 
breast cancer (BC) have been found to be as predictive of these diseases as well known monogenic mutations ${ }^{11}$, which tend to be rarer, and could lead to improved screening for larger numbers of individual who are at risk. Individuals in the top $5 \%$ of the PRS distributions had $\sim 3$ fold likelihood of having CAD, AF, T2D, IBS, or BC compared to the bottom 95\%. For obesity, individuals in the top PRS decile were on average $13 \mathrm{~kg}$ heavier than those in the bottom decile ${ }^{12}$. These studies demonstrate the potential for identifying individuals at heightened risk for various medical conditions using PRS. Given that AUD is a moderately heritable trait and GWAS for alcohol-related phenotypes are beginning to identify numerous variants associated with these outcomes, PRS for alcohol-related outcomes may be also able to identify individuals at heightened risk of developing an AUD.

In the current analysis, we tested PRS in two target samples, a population-based sample and a clinically ascertained sample of families deeply affected by AUD, to evaluate the current state of alcohol-related PRS in relation to AUD and identifying those at heightened risk. We use several discovery samples from large-scale GWAS to create three PRS: a meta-analysis of two GWASs on alcohol-related problems ${ }^{5,6}$, a recent largescale GWAS of alcohol consumption ${ }^{8}$, and a GWAS for risky behaviors, including alcohol use ${ }^{13}$. We chose to test PRS based on multiple alcohol-related GWAS because multiple lines of evidence indicate alcohol consumption and dependence have only partially shared genetic etiology $5,6,14,15$. Additionally, we include a PRS for general risk behavior as there is robust evidence that the genetic risk for alcohol and other substance use disorders is shared with other disorders and behaviors related to reduced inhibitory control ${ }^{16-18}$. Similar to recent work for specific medical conditions ${ }^{11}$, we compare the upper end of the PRS distribution at various thresholds (top 20,10, and 5\%) to examine whether focusing on these upper parts of the distribution provide additional information in identifying those at increased risk of developing an AUD. We acknowledge the exploratory nature of these analyses and the arbitrary nature of our thresholds in the absence of well-defined clinical risk scores, such as those for medical conditions like hypertension. Finally, we test the association of these PRSs with other substance use disorders (including nicotine and illicit substance use disorders), based on the robust finding that substance use disorders share an underlying genetic architecture, with the majority of the heritability shared across substances ${ }^{16-18}$.

\section{Methods \\ Samples}

The FinnTwin12 Study (FT12) is a population-based study of Finnish twins born 1983-1987 identified through Finland's Central Population Registry. A total of 2705 families (87\% of all identified) returned the initial family questionnaire late in the year in which twins reached age 11. Twins were invited to participate in follow-up surveys when they were ages 14, 17, and approximately 22 (during young adulthood). An intensively studies sample was selected as 1035 families, among whom 1854 twins were interviewed at age 14 . The interviewed twins were invited as young adults to complete the Semi-Structured Assessment for the Genetics of Alcoholism (SSAGA) $)^{19,20}$ interview $(n=$ 1,347 ) and provide DNA samples (see Kaprio 2013 for a full description). The current analysis uses data from the young adult wave (mean age $=21.9$; range $20-26$ ), which included retrospective lifetime diagnoses.

The Collaborative Study on the Genetics of Alcoholism (COGA) is a sample of high-risk families ascertained through adult probands in treatment for AUD and a smaller set of comparison families from the same communities. In the first 10 years, probands along with all willing first-degree relatives were assessed; recruitment was extended to include additional relatives. Data collection included the SSAGA $^{19}$, neurophysiological and neuropsychological protocols, and collection of blood for DNA. In 2004, COGA began a prospective study of adolescents and young adults, targeting assessment of youth aged 12-22 from COGA families where at least one parent had been interviewed. These young participants were re-assessed every two years. The sample is racially/ethnically diverse $(60.6 \%$ nonHispanic White, 24.9\% Black, 11.1\% Hispanic, and 3.4\% other). Most (84\%) have GWAS data. A full description of the COGA sample is available elsewhere ${ }^{21-23}$. For the present study, we only focused on COGA participants of empirically assigned (as verified from GWAS data) European ancestry $(n=7599)$ because each of the discovery GWAS samples were primarily of European ancestry. Ancestral mismatch between discovery and target samples can lead to bias in the performance of polygenic scores $^{24}$.

\section{Measures \\ Alcohol use disorder (AUD)}

We used SSAGA interviews to construct lifetime criteria counts of DSM-5 AUD ${ }^{25}$ in each sample. Because individuals in COGA are potentially interviewed multiple times, we used the highest criteria count ever reported by each subject. In FT12, lifetime criteria counts were measured at the young adult interview. In addition to criteria counts, we created AUD thresholds for those who met criteria for mild ( $2+$ criteria), moderate $(4+$ criteria), or severe $\left(6+\right.$ criteria) AUD $^{25}$ without clustering. In both FT12 and COGA, individuals who had never initiated alcohol use were coded as missing. 


\section{Other substance use disorders (SUD)}

We constructed lifetime criteria counts of cannabis, cocaine, and opioid use disorders based on DSM-5 criteria. We measured nicotine dependence criteria using the Fagerstrom Test for Nicotine Dependence (FTND), which assesses six criteria and has values ranging from 0 to 10 in both COGA and FT12. Because many illicit SUDs were not measured or rare in the FT12 data, we limit analyses of illicit SUD to COGA. Like AUD, these criteria counts represent the maximum reported for each respondent across the course of the study. Criteria counts for each substance were limited to those who indicated ever using the corresponding substance. In the case of FTND, this is limited to those who report smoking $100+$ cigarettes in their lifetime.

\section{Polygenic scores (PRS)}

We created PRS derived from publicly available largescale GWASs. Information on genotyping and quality control is available in the Supplementary information (Section 1). We created PRS using a Bayesian regression and continuous shrinkage method (PRS-CS) ${ }^{26}$. PRS-CS uses LD information from an external reference panel (1000 Genomes Phase III European subsample) to estimate the posterior effect sizes for each SNP in a given set of GWAS summary statistics. Both empirical tests and simulations have shown improved predictive power above traditional methods of score construction ${ }^{26}$. For computational purposes, we limited the SNPS for score creation to HapMap3 SNPs that overlapped between the original GWAS summary statistics, the LD reference panel, and the target samples for score creation. We converted PRS to Z-scores for interpretation.

We used four primary discovery GWASs to create three different PRSs. The first was from a recent GWAS of number of alcoholic drinks per week in approximately one million individuals provided by the GWAS \& Sequencing Consortium of Alcohol and Nicotine Use $(\text { GSCAN })^{8}$. We obtained GSCAN summary statistics with all Finnish (which included FinnTwin12) and 23andMe (which are not publicly available) cohorts removed (available $N=534,683$ ). The PRS for alcohol problems were derived from a meta-analysis of two GWASs: a GWAS on the problem subscale from the Alcohol Use Disorders Identification Test (questions 4-10; AUDIT-P) in 121,604 individuals from the UK Biobank ${ }^{6}$ and the Psychiatric Genomcs Consurtium's (PGC) GWAS of alcohol dependence $(N=46,568)^{5}$. Both FT12 and COGA were in the initial AD GWAS and we obtained summary statistics with each cohort removed (meta-analysis results available in supplemental info Tables S1, S2 and Figs. S1, S2). Finally, we derived a PRS for risky behaviors from a GWAS of the first prinicipal component of four risky behaviors (drinks per week, ever smoking, propensity for driving over the speed limit, and number of sexual partners) from 315,894 individuals in the UK Biobank $(\mathrm{UKB})^{13}$. While this PRS does include alcohol consumption and smoking, it captures the shared variance between these substance use measures and the other two risky behaviors. These polygenic scores covered the domains of alcohol consumption (GSCAN DPW), alcohol problems (PROB ALC), and general externalizing (RISK PC).

\section{Analytic strategy}

We first identified the predictive power for each PRS in both COGA and FT12 using the change in $\mathrm{R}^{2}$ above a baseline model with sex, age of last observation, the first ten ancestral principal components (PCs), genotyping array, and data collection site (these latter two were only included in COGA analyses). We used linear/generalized-linear mixed-effects models with random intercepts to adjust for clustering at the family level and a pseudo- $R^{2}$ for mixed models ${ }^{27}$. In addition to the predictive power of individual PRS, we estimated the conditional effect of all PRS on AUD criteria to examine whether each PRS explained unique variance in AUD criteria. We also calculated the area under the curve (AUC) of the conditional model containing all continuous PRS to estimate sensitivity/specificity ${ }^{28}$. AUC provides an estimate of the probability a randomly selected case has predicted value more extreme than that of a randomly chosen control ${ }^{29}$. An AUC of 0.5 indicates that a classifier does not provide any useful information in determining cases from controls (see supplemental information Section 3). We next divided PRSs at several thresholds (80th, 90th, and 95th percentiles) to examine whether there was a non-linear increase in risk of AUD (using symptom severity thresholds of AUD) across the PRS continuum. Finally, we compared mean values of other substance use outcomes for the top 5\% in each PRS to those in the bottom 95\%. We selected this threshold based in the increased prevalence of AUD in those in the top 5\% of the PRS distributions (see Fig. S3). All code is available upon request from the corresponding author.

\section{Results}

Table 1 presents the descriptive statistics for each of the samples. Each sample has slightly more female than male participants. The mean number of AUD criteria (3.44) in COGA was relatively high, as COGA was primarily ascertained for families with multiple AUD members. COGA participants report a mean of 4.17 for FTND criteria. For other SUD criteria in COGA, there are a substantial number of participants who report non-zero levels of criteria, though criteria counts for cannabis, cocaine, and opioid use disorders are zero-inflated (see Table 1). The mean number of AUD and FTND criteria in 
Table 1 Descriptive Statistics for FT12 and COGA samples.

\begin{tabular}{|c|c|c|c|c|c|c|c|c|}
\hline Sample & & $N$ & Mean/\% & Median & $\% 0$ & SD & Min & Max \\
\hline \multirow[t]{7}{*}{ COGA } & Female & 7599 & $52.84 \%$ & - & - & - & - & - \\
\hline & Age & 7599 & 36.94 & - & - & 14.77 & 12 & 91 \\
\hline & DSM-5 AUD criteria & 7300 & 3.44 & 2 & $28.79 \%$ & 3.63 & 0 & 11 \\
\hline & DSM-5 CUD criteria & 5051 & 2.37 & 1 & $48.19 \%$ & 3.13 & 0 & 11 \\
\hline & DSM-5 CoUD criteria & 2404 & 3.18 & 0 & $50.17 \%$ & 4.13 & 0 & 11 \\
\hline & DSM-5 OUD criteria & 1663 & 2.05 & 0 & $62.96 \%$ & 3.51 & 0 & 11 \\
\hline & FTND count & 3701 & 4.12 & 4 & $14.02 \%$ & 2.74 & 0 & 10 \\
\hline \multirow[t]{4}{*}{ FT12 } & Female & 1251 & $54.40 \%$ & - & - & - & - & - \\
\hline & Age & 1247 & 21.94 & - & - & 0.77 & 21 & 26 \\
\hline & DSM-5 AUD criteria & 1215 & 1.63 & 1 & $34.57 \%$ & 1.84 & 0 & 11 \\
\hline & FTND count & 631 & 2.57 & 2 & $21.55 \%$ & 2.13 & 0 & 10 \\
\hline
\end{tabular}

The $N$ reflects those who report lifetime ever use of that substance. All criteria counts limited to individuals who had initiated use of that substance. The $\% 0$ represents the percentage of participants who have initiated use and have no reported criteria.

AUD alcohol use disorder, CUD cannabis use disorder, CoUD cocaine use disorder, OUD opioid use disorder, FTND Fagerstrom test for nicotine dependence (limited to those who report ever smoking 100 cigarettes).

the population-based FT12 sample were 1.63 and 2.57, respectively.

\section{Predictive power of PRS}

Across each sample and PRS, we observed significant associations between PRS and AUD criteria, even after correcting for a false discovery rate $(\mathrm{FDR})^{30}$ of $5 \%$. In COGA the GSCAN DPW PRS was most strongly associated with AUD criteria $\left(\Delta R^{2}=0.017\right)$, followed closely by the RISK PC PRS $\left(\Delta \mathrm{R}^{2}=0.016\right)$, and lastly the PROB ALC PRS $\left(\Delta R^{2}=0.012\right)$. We see a similar pattern in FT12, where the GSCAN DPW PRS was the strongest association $\left(\Delta \mathrm{R}^{2}=0.030\right)$, the RISK PC PRS was slightly weaker $\left(\Delta \mathrm{R}^{2}=0.023\right)$, and the PROB ALC PRS performed the worst $\left(\Delta \mathrm{R}^{2}=0.001\right)$.

Next, we determined whether each of these PRS contributed to AUD criteria in a model containing all three, simultaneously. Figure 1 presents the parameter estimates for the independent and conditional effect of each PRS in both COGA and FT12. In the conditional model for COGA, each of the PRSs remains significantly associated with AUD criteria, though the associations are attenuated (conditional model $\Delta \mathrm{R}^{2}=0.025$ ). In FT12, the PRS for RISK PC and GSCAN DPW remain significant in the conditional model, while the association for PROB ALC PRS is no longer significant (conditional model $\Delta \mathrm{R}^{2}=$ 0.035). We averaged the three PRS into one composite PRS score in COGA and averaged the RISK PC and GSCAN DPW PRS in FT12 to carry forward in the following analyses.

Finally, we assessed the sensitivity/specificity of these combined PRS by calculating the AUC from receiver operating characteristic (ROC) curves, presented in Fig. 2. AUC from the full model (including both continuous PRS and covariates) for each level of AUD severity ranged from 0.67 to 0.74 in COGA and from 0.65 to 0.76 in FT12. Comparing the AUC for the models with and without PRSs, including the PRS only provided a slight increase in AUC.

\section{Increase in risk across the polygenic continuum}

In order to estimate whether individuals at the extreme end of the PRS distribution were at elevated risk of AUD, we compared the risk of AUD between those above and below a given threshold in the distribution. We divided these PRSs at the 80th, 90th, and 95th percentile in each sample and estimated the odds ratio (OR) for AUD in the top portion of the distribution relative to the bottom portion of the distribution (e.g., splitting at the 80th percentile compares the top $20 \%$ to the bottom $80 \%$ ). Table 2 provides the estimates for all of those models. Across each threshold for AUD severity in COGA, we observed a similar pattern where, as expected, those in the upper end of the polygenic distribution had greater odds of meeting criteria for AUD. However, regardless of the threshold, the OR's at each threshold were roughly equivalent. For example, in the case of severe AUD, when dividing 80th percentile $(\mathrm{OR}=1.98 ; 95 \% \mathrm{CI}=1.53$, 2.57), 90th percentile $(\mathrm{OR}=2.02 ; 95 \% \mathrm{CI}=1.73,2.36)$, or 95 th percentile $(\mathrm{OR}=1.96 ; 95 \% \mathrm{CI}=1.59,2.40)$, all of confidence intervals for the point estimates overlap. In FT12, there was a similar pattern. Though some of the point estimates appear to increase as the thresholds become more restrictive, the confidence intervals again overlap. 


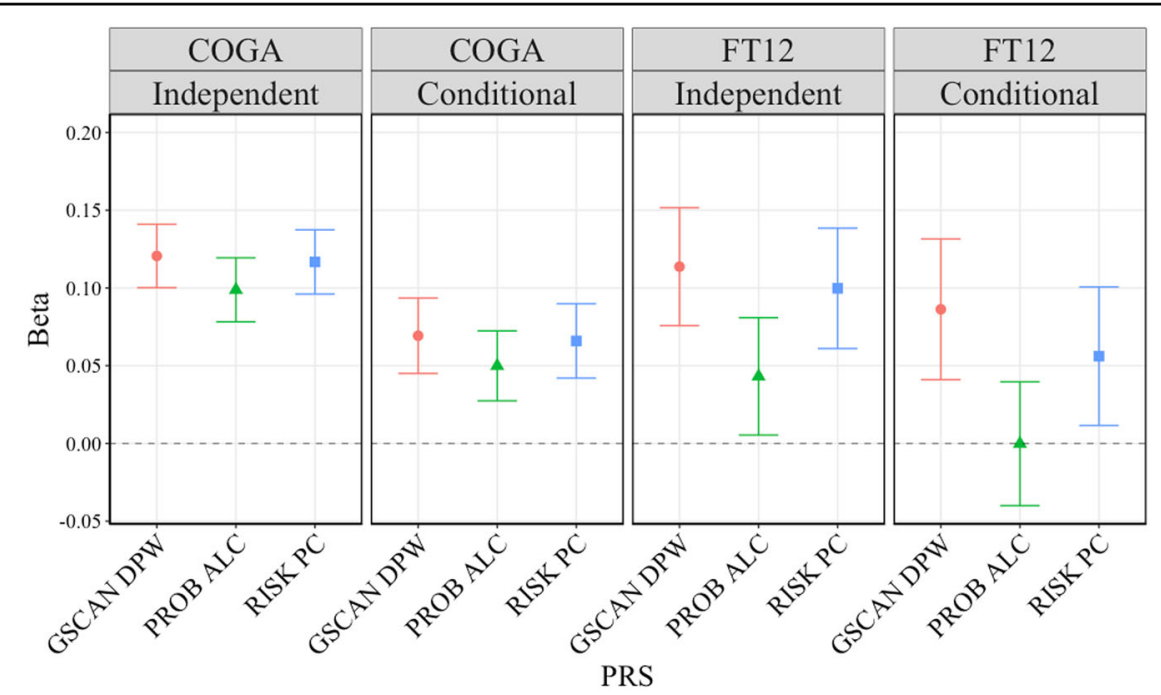

Fig. 1 Parameter estimates for PRS in independent and conditional models. Parameter estimates (with 95\% confidence intervals), from linear mixed models for alcohol use disorder (AUD) criteria regressed on polygenic risk scores (PRS) for drinks per week (GSCAN DPW), problem alcohol use (PROB ALC), and risky behaviors (RISK PC) in COGA and FT12. Independent = model with only corresponding PRS. Conditional = model with all PRS included. Adjusted for age, sex, first 10 ancestral principal components, genotyping array, and data collection site (only COGA for the latter two). All tests were two-sided.
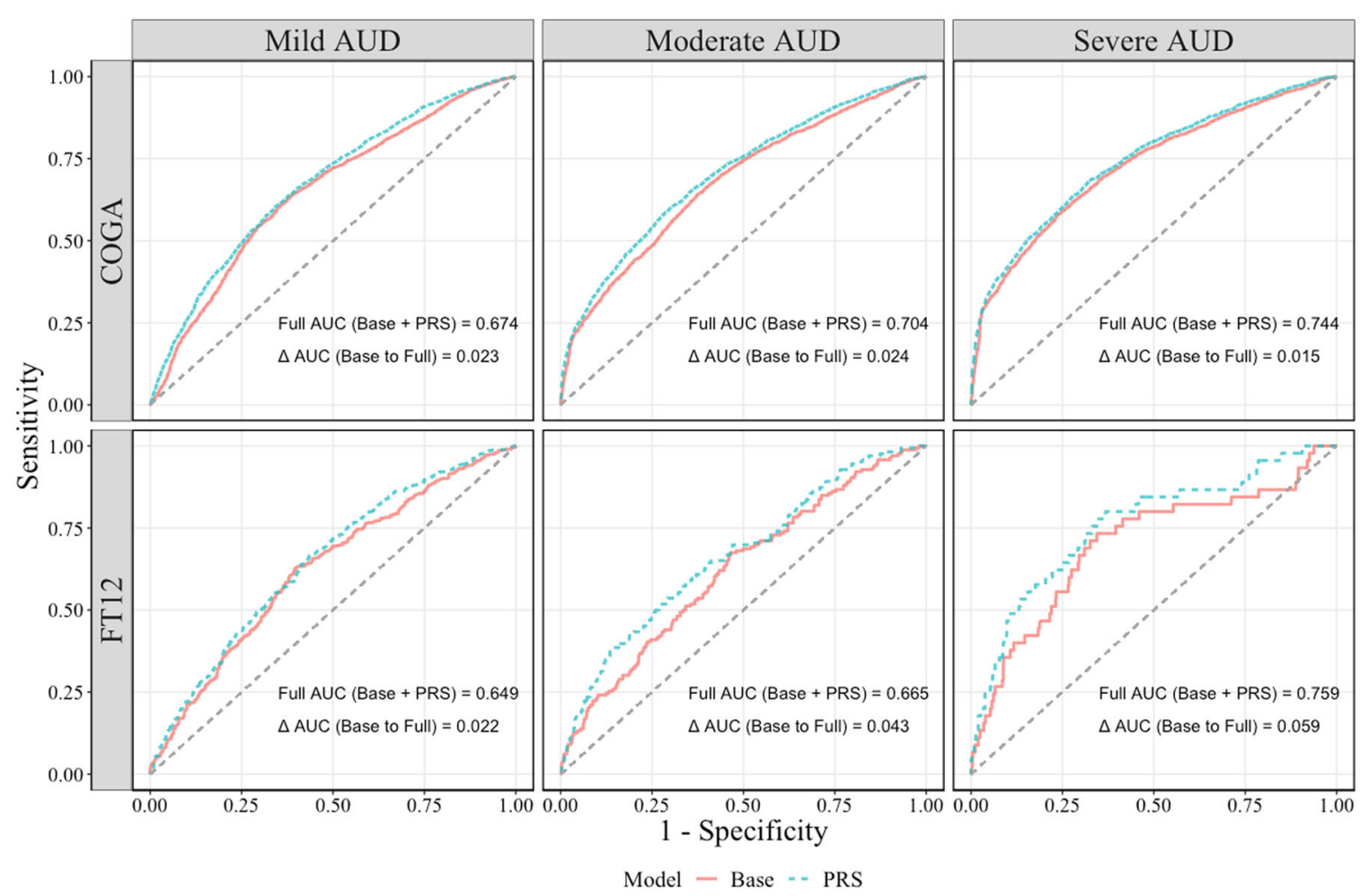

Fig. 2 ROC curves for baseline and PRS models. Receiver operating characteristic (ROC) curves for baseline models (covariates only) and polygenic risk score (PRS) models (PRS + covariate) for each level of severity in alcohol use disorder (AUD). Area under the curve (AUC) for the PRS model (Full $A \cup C$ ) and change in in AUC from Base to PRS model $\triangle A \cup C$ ) is presented each cell. AUC provides an estimate of the probability a randomly selected subject with the condition has a test result indicating greater suspicion than that of a randomly chosen subject without the condition ${ }^{29}$. An AUC of 0.5 indicates that a classifier does not provide any useful information in determining cases from controls. 
Table 2 Odds ratios for those at extreme end of the PRS continuum.

\begin{tabular}{|c|c|c|c|c|c|c|c|}
\hline Sample & Phenotype & Prevalence & Split & $N$ Cases & OR & 95 \% Cl Low & $95 \% \mathrm{Cl}$ High \\
\hline & Mild AUD & $57.06 \%$ & $80 \%$ & 998 & $1.96^{*}$ & 1.70 & 2.26 \\
\hline \multirow[t]{3}{*}{ COGA } & Mild AUD & $57.06 \%$ & $90 \%$ & 501 & $1.81^{*}$ & 1.49 & 2.19 \\
\hline & Mild AUD & $57.06 \%$ & $95 \%$ & 258 & $1.89^{*}$ & 1.45 & 2.47 \\
\hline & Moderate AUD & $37.44 \%$ & $80 \%$ & 738 & $2.07^{*}$ & 1.79 & 2.38 \\
\hline \multirow[t]{3}{*}{ COGA } & Moderate AUD & $37.44 \%$ & $90 \%$ & 383 & $1.94^{*}$ & 1.60 & 2.34 \\
\hline & Moderate AUD & $37.44 \%$ & $95 \%$ & 201 & $1.98^{*}$ & 1.53 & 2.57 \\
\hline & Severe AUD & $25.89 \%$ & $80 \%$ & 534 & $2.02^{*}$ & 1.73 & 2.36 \\
\hline \multirow[t]{3}{*}{ COGA } & Severe AUD & $25.89 \%$ & $90 \%$ & 285 & $1.96^{*}$ & 1.59 & 2.40 \\
\hline & Severe AUD & $25.89 \%$ & $95 \%$ & 146 & $1.81^{*}$ & 1.38 & 2.39 \\
\hline & Mild AUD & $41.98 \%$ & $80 \%$ & 123 & $1.78^{*}$ & 1.21 & 2.61 \\
\hline \multirow[t]{3}{*}{ FT12 } & Mild AUD & $41.98 \%$ & $90 \%$ & 68 & $2.35^{*}$ & 1.41 & 3.93 \\
\hline & Mild AUD & $41.98 \%$ & $95 \%$ & 32 & 1.94 & 0.97 & 3.88 \\
\hline & Moderate AUD & $13.91 \%$ & $80 \%$ & 55 & $2.85^{*}$ & 1.72 & 4.74 \\
\hline \multirow[t]{3}{*}{ FT12 } & Moderate AUD & $13.91 \%$ & $90 \%$ & 32 & $3.50^{*}$ & 1.85 & 6.64 \\
\hline & Moderate AUD & $13.91 \%$ & $95 \%$ & 15 & $3.14^{*}$ & 1.33 & 7.42 \\
\hline & Severe AUD & $3.79 \%$ & $80 \%$ & 16 & $2.84^{*}$ & 1.37 & 5.87 \\
\hline \multirow[t]{2}{*}{ FT12 } & Severe AUD & $3.79 \%$ & $90 \%$ & 12 & $4.41^{*}$ & 2.04 & 9.54 \\
\hline & Severe AUD & $3.79 \%$ & $95 \%$ & 5 & 2.98 & 0.96 & 9.30 \\
\hline
\end{tabular}

All models control for sex, age at last interview, and first 10 principal components. Models for COGA also included data collection site and genotyping array. $N$ Cases $=$ number of individuals who meet criteria for a given level of AUD and are in the top portion of the split.

${ }^{*} p<0.05$ (two-sided) after correcting for $5 \%$ false discovery rate (FDR).

Examining the substance use phenome of the extreme end of the polygenic risk continuum

We compared the likelihood of substance-related outcomes in individuals in the top $5 \%$ of each of the PRS in COGA and FT12 (adjusted for covariates). Figure 3 presents the mean lifetime criteria endorsed for a variety of substance use disorders (alcohol, cannabis, cocaine, nicotine, and opioid) for individuals in the top 5\% for each PRS relative to the bottom 95\% of each PRS. In COGA, individuals in the top $5 \%$ of the PROB ALC, RISK PC, and/or GSCAN DPW PRS had significantly higher levels of alcohol (0.25-0.31 SD), while individuals in the top $5 \%$ of the PROB ALC and RISK PC had higher mean nicotine criteria (0.10-0.16 SD) than those in the bottom 95\% of the PRS distribution. Those in the top 5\% of the RISK PC PRS also endorsed a higher number of criteria for cannabis use disorder (0.14 SD) and opioid use disorder (0.19 SD). In FT12, those in the top $5 \%$ in the top $5 \%$ of the RISK PC and GSCAN DPW PRS had significantly higher levels of AUD criteria (0.25-0.31 SD) but did not differ on FTND criteria.

Overall, individuals in the top $5 \%$ of any PRS report greater levels of any substance, though being in the top 5\% of the RISK PC PRS is associated with the most other substances. These PRS are modestly correlated with one another in both COGA $\left(r_{R I S K ~ P C * P R O B ~ A L C}=0.34 ; r_{G S C A N}\right.$ $\left.D P W^{*} R I S K \quad P C=0.49 ; r_{G S C A N} D P W^{*} P R O B \quad A L C=0.40\right)$ and

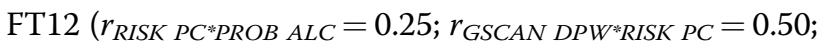
$\left.r_{G S C A N D P W^{*} P R O B A L C}=0.35\right)$, but each captures unique information related to the genetics of substance use problems (and other risky behaviors).

\section{Discussion}

Researchers have begun to evaluate the potential for use of PRS (for a variety of medical phenotypes) ${ }^{11,12}$ in clinical settings. In this analysis, we examined the current predictive power and strength of association between several PRSs and a variety of SUDs, with a focus on AUD in both a clinically ascertained and a population-based sample. We were interested in (1) which scores based on available GWASs provided the strongest association with alcohol use disorder, whether these scores explained unique variance in AUD in a conditional model, and how well these scores discriminated between cases and controls; (2) what the risk of AUD was for those at the upper end of the risk continuum compared to the bottom; and 3) the levels of substance use disorder criteria for individuals at the top $5 \%$ of the polygenic score continuum compared to remaining $95 \%$. 


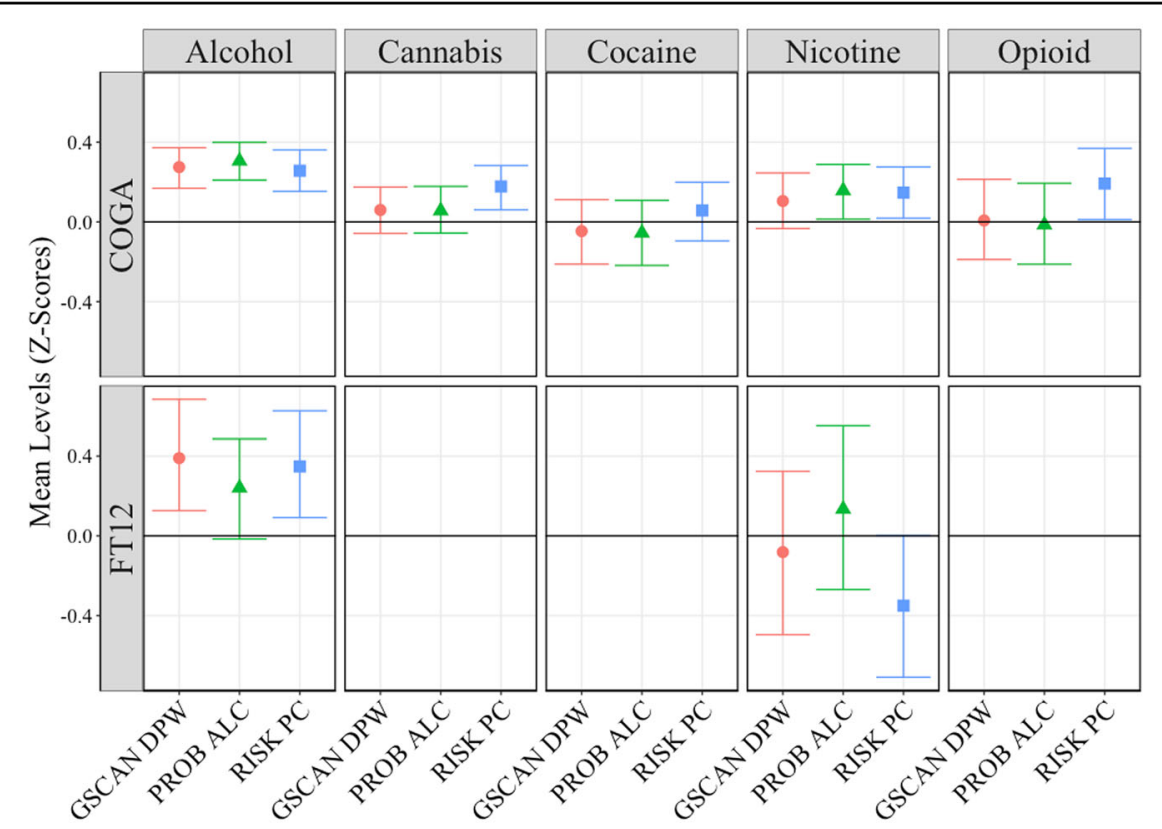

Fig. 3 Top 5\% of PRS Continuum. Mean levels of substance use disorder (SUD) criteria for alcohol, cannabis, cocaine, nicotine, and opioid use disorders for top $5 \%$ of each polygenic risk score (PRS) compared to the bottom 95\%. Black bar represents mean of bottom $95 \%$ of each sample. $95 \%$ confidence intervals estimated using 1000 bootstrap resampling.

In terms of which polygenic scores were the most predictive, we considered three scores: one based on problematic alcohol use (PROB ALC), one based on alcohol consumption (GSCAN DPW), and one based on general risky behaviors (RISK PC), as twin and family studies have shown alcohol and other risk behaviors to be genetically correlated traits ${ }^{6,14-18}$. In both samples, the GSCAN DPW PRS was the most strongly associated, followed closely by the RISK PC PRS. When we included all of the PRS in one model, all three PRS were associated with AUD criteria in COGA. Only the RISK PC and GSCAN DPW PRS were associated with AUD criteria in FT12. Overall, the unique contributions of each PRS reinforce the notion that the genetics of AUD are multifaceted, comprised of risk for level of consumption, alcohol-related problems, and behavioral disinhibition ${ }^{31,32}$. Evaluating the AUC for the combined PRS revealed the combined effect of PRSs only marginally improved the AUC, similar to recent analyses for coronary artery disease $\mathrm{s}^{33}$ and ischemic stroke ${ }^{34}$. We ran a series of sensitivity analyses to test whether differences across the samples reflected age differences rather than differences in ascertainment. Restricting COGA to participants under 30 did not fundamentally change the results (see Supplementary information Section 4, Table S3, and Fig. S4). Evaluating the AUC for the combined PRS revealed the combined effect of PRSs only marginally improved the AUC over models with just covariates.

In an exploratory approach, we chose a series of more restrictive thresholds to divide the PRS distribution. The odds of having an AUD were statistically indistinguishable across each of the thresholds in both COGA and FT12. Even though the point estimates increased in some cases, the confidence intervals around these estimates were relatively large and they did not differ significantly. Additionally, there were only a small number of individuals in the severe category in FT12 and we urge caution in interpreting these estimates. Finally, the top $5 \%$ of the continuum for each PRS reported elevated rates of other SUD criteria (cannabis, cocaine, opioid, and nicotine use disorders) compared to the bottom 95\%. The RISK PC PRS was most associated with higher mean levels of SUD criteria, suggesting that risk for externalizing may be particularly useful in identifying individuals at risk for multiple SUDs.

These initial findings suggest the current PRSs are unlikely to prove useful for SUDs in a clinical setting. Being able to eventually identify those at heightened risk for SUDs may allow for more targeted early intervention and prevention. However, before this is possible, larger discovery GWAS across substance use phenotypes with PRS that explain greater portions of the variance will be necessary. As GWAS sample sizes for SUDs increase, we will likely see increases in effect sizes ${ }^{35}$. Additionally, using multivariate techniques to model the shared genetic architecture across existing SUD GWAS to include both aspects of externalizing and internalizing (e.g. depression, anxiety) may also improve prediction $^{36,37}$. Inclusion of genetic data in a clinical setting will also require that psychiatrists and clinicians receive greater training in genetics and/or that they partner with genetic 
counselors, so they are both better able to understand what increased genetic risk means and be able convey that information accurately to their patients ${ }^{38,39}$. In addition to clinical utility, we must ensure that regulations and protections surrounding the use of genetic information in clinical settings can adequately protect the rights of individuals who are identified to be "at risk."

This research has several important limitations. First, all analyses were limited to individuals of European ancestry because the discovery GWASs available were conducted in individuals of primarily European ancestry. It will be important to ascertain sizable samples of subjects with nonEuropean ancestries to properly estimate the predictive utility of PRS in non-European samples. This is especially important for racial-ethnic minorities so that health disparities are not further perpetuated ${ }^{40}$. Second, our use of lifetime diagnoses may obscure the impact of changing genetic influences on the development of AUD across the life course ${ }^{41,42}$. Future work should draw on longitudinal data to examine the ways in which the strength of associations for PRS changes with the age of the target sample. Third, UKB was a large portion of the discovery sample for each of the GWAS used to create PRS. To the degree that UKB is biased, each of the PRS in these analyses will also reflect that bias ${ }^{43}$. Finally, these analyses examined the marginal influence of PRS, independent of environment. Processes of gene-environment interaction (GxE) are well documented in alcohol misuse ${ }^{44-47}$. Incorporating environmental information along with PRS in a methodologically rigorous manner will be an important next step in developing clinically predictive algorithms.

Polygenic scores are becoming better powered and starting to explain non-trivial portions of variance. We examined the current state of PRS for substance use, with a focus on AUD. Each of the PRSs analyzed here were associated with AUD. However, the maximum variance explained by any single score was still small ( 2\%). Individuals at the top of the PRS continuum had elevated rates of multiple substance use problems, but these differences across the PRS continuum are unlikely to be of broad clinical use in their current state. As GWAS discovery samples become larger and we are better able to model the complex relationship between genotype and phenotype, polygenic scores may eventually be useful in a clinical setting.

\footnotetext{
Acknowledgements

Research reported in this publication was supported by the National Institute on Alcohol Abuse and Alcoholism of the National Institutes of Health under award numbers R01AA015416 (D.M.D.), K02AA018755 (D.M.D.), K02DA32573 (A.A.), K01DA037914 (J.L.M.), and F32AA027435 (E.C.J.); the Academy of Finland (grants 100499, 205585, 118555, 141054, 265240, 308248, 308698 and 312073); and the Scientific and Technological Research Council of Turkey (TÜBITAK) under award number 114C117 (F.A.). The content is solely the responsibility of the authors and does not necessarily represent the official views of the National Institutes of Health, the Academy of Finland, or the Scientific and Technological Research Council of Turkey. This research also used summary data from the Psychiatric Genomics Consortium (PGC) Substance Use Disorders (SUD) working group. The PGC-SUD is
}

supported by funds from NIDA and NIMH to MH109532 and, previously, had analyst support from NIAAA to U01AA008401 (COGA). PGC-SUD gratefully acknowledges its contributing studies and the participants in those studies, without whom this effort would not be possible.

\section{COGA}

The Collaborative Study on the Genetics of Alcoholism (COGA), Principal Investigators B. Porjesz, V. Hesselbrock, H. Edenberg, L. Bierut, includes eleven different centers: University of Connecticut (V. Hesselbrock); Indiana University (H.J. Edenberg, J. Nurnberger Jr., T. Foroud; Y. Liu); University of lowa (S. Kuperman, J. Kramer); SUNY Downstate (B. Porjesz); Washington University in St. Louis (L. Bierut, J. Rice, K. Bucholz, A. Agrawal); University of California at San Diego (M. Schuckit); Rutgers University (J. Tischfield, A. Brooks); Department of Biomedical and Health Informatics, The Children's Hospital of Philadelphia; Department of Genetics, Perelman School of Medicine, University of Pennsylvania, Philadelphia PA (L. Almasy), Virginia Commonwealth University (D. Dick), Icahn School of Medicine at Mount Sinai (A. Goate), and Howard University (R. Taylor). Other COGA collaborators include: L. Bauer (University of Connecticut); J. McClintick, L. Wetherill, X. Xuei, D. Lai, S. O'Connor, M. Plawecki, S. Lourens (Indiana University); G. Chan (University of lowa; University of Connecticut); J. Meyers, D. Chorlian, C. Kamarajan, A. Pandey, J. Zhang (SUNY Downstate); J.C. Wang, M. Kapoor, S. Bertelsen (Icahn School of Medicine at Mount Sinai); A. Anokhin, V. McCutcheon, S. Saccone (Washington University); J. Salvatore, F. Aliev, B. Cho (Virginia Commonwealth University); and Mark Kos (University of Texas Rio Grande Valley). A. Parsian and H. Chen are the NIAAA Staff Collaborators.

We continue to be inspired by our memories of Henri Begleiter and Theodore Reich, founding PI and Co-PI of COGA, and also owe a debt of gratitude to other past organizers of COGA, including Ting-Kai Li, P. Michael Conneally, Raymond Crowe, and Wendy Reich, for their critical contributions. This national collaborative study is supported by NIH Grant U10AA008401 from the National Institute on Alcohol Abuse and Alcoholism (NIAAA) and the National Institute on Drug Abuse (NIDA).

\section{Author details}

'Department of Psychology, Virginia Commonwealth University, Richmond, VA, USA. ${ }^{2}$ Department of Health Behavior and Policy, Virginia Commonwealth University, Richmond, VA, USA. ${ }^{3}$ Department of Psychology, Arizona State University, Tempe, AZ, USA. ${ }^{4}$ Department of Psychiatry, School of Medicine, Washington University in St. Louis, St Louis, MO, USA. ${ }^{5}$ Department of Psychiatry \& Behavioral Sciences, State University of New York Downstate Medical Center, Brooklyn, NY, USA. ${ }^{6}$ Department of Medical and Molecular Genetics, School of Medicine, Indiana University, Indianapolis, IN, USA.

${ }^{7}$ Institute for Molecular Medicine Finland, University of Helsinki, Helsinki, Finland. ${ }^{8}$ Institute of Criminology and Legal Policy, University of Helsinki, Helsinki, Finland. ${ }^{9}$ Faculty of Business, Karabük University, Karabük, Turkey. ${ }^{10}$ Department of Psychiatry, School of Medicine, University of Connecticut, Farmington, CT, USA. " Department of Psychiatry, Carver College of Medicine, University of lowa, lowa City, IA, USA. ${ }^{12}$ Department of Psychiatry, School of Medicine, Indiana University, Indianapolis, IN, USA. ${ }^{13}$ Stark Neurosciences Research Institute, School of Medicine, Indiana University, Indianapolis, IN, USA. ${ }^{14}$ Department of Psychological and Brain Sciences, Indiana University, Bloomington, IN, USA. ${ }^{15}$ Department of Biochemistry and Molecular Biology, School of Medicine, Indiana University, Indianapolis, IN, USA. ${ }^{16}$ Department of Psychiatry, School of Medicine, University of California, San Diego, San Diego, CA, USA. ${ }^{17}$ Department of Public Health, University of Helsinki, Helsinki, Finland. ${ }^{18}$ Department of Human and Molecular Genetics, School of Medicine, Virginia Commonwealth University, Richmond, VA, USA

\section{Conflict of interest}

Dr. John Nurnberger is an investigator for Janssen.

\section{Publisher's note}

Springer Nature remains neutral with regard to jurisdictional claims in published maps and institutional affiliations.

Supplementary Information accompanies this paper at (https://doi.org/ 10.1038/s41398-020-00865-8). 
Received: 13 September 2019 Revised: 22 May 2020 Accepted: 26 May 2020

Published online: 18 June 2020

\section{References}

1. Gakidou, E. et al. Global, regional, and national comparative risk assessment of 84 behavioural, environmental and occupational, and metabolic risks or clusters of risks, 1990-2016: a systematic analysis for the Global Burden of Disease Study 2016. Lancet 390, 1345-1422 (2017).

2. World Health Organization. Global Status Report on Alcohol and Health (Switzerland, Geneva, 2018).

3. Mokdad, A. H. et al. The State of US Health, 1990-2016: burden of diseases, injuries, and risk factors among US states. JAMA 319, 1444-1472 (2018).

4. Verhulst, B., Neale, M. C. \& Kendler, K. S. The heritability of alcohol use disorders: a meta-analysis of twin and adoption studies. Psychological Med. 45, 1061-1072 (2015).

5. Walters, R. K. et al. Transancestral GWAS of alcohol dependence reveals common genetic underpinnings with psychiatric disorders. Nat. Neurosci. 21, 1656-1669 (2018).

6. Sanchez-Roige, S. et al. Genome-wide association study meta-analysis of the alcohol use disorders identification test (AUDIT) in two population-based cohorts. Am. J. Psychiatry 176, 107-118 (2019).

7. Kranzler, H. R. et al. Genome-wide association study of alcohol consumption and use disorder in 274,424 individuals from multiple populations. Nat. Commun. 10, 1499 (2019).

8. Liu, M. et al. Association studies of up to 1.2 million individuals yield new insights into the genetic etiology of tobacco and alcohol use. Nat. Genet. 51, 237-244 (2019).

9. Gelernter, J. et al. Genomewide assoÿciation study of maximum habitual alcohol intake in $>140,000$ US European- and African-American veterans yields novel risk loci. Biol. Psychiatry 86, 365-376 (2019).

10. Pasman, J. A. et al. GWAS of lifetime cannabis use reveals new risk loci, genetic overlap with psychiatric traits, and a causal influence of schizophrenia. Nat. Neurosci. 21, 1161-1170 (2018).

11. Khera, A. V. et al. Genome-wide polygenic scores for common diseases identify individuals with risk equivalent to monogenic mutations. Nat. Genet. 50, 1219-1224 (2018).

12. Khera, A. V. et al. Polygenic prediction of weight and obesity trajectories from birth to adulthood. Cell 177, 587-596 e589 (2019).

13. Karlsson Linnér, R. et al. Genome-wide association analyses of risk tolerance and risky behaviors in over 1 million individuals identify hundreds of loci and shared genetic influences. Nat. Genet. 51, 245-257 (2019).

14. Dick, D. M., Meyers, J. L., Rose, R. J., Kaprio, J. \& Kendler, K. S. Measures of current alcohol consumption and problems: two independent twin studies suggest a complex genetic architecture. Alcohol.: Clin. Exp. Res. 35, 2152-2161 (2011).

15. Kendler, K. S., Myers, J., Dick, D. \& Prescott, C. A. The relationship between genetic influences on alcohol dependence and on patterns of alcohol consumption. Alcohol. Clin. Exp. Res. 34, 1058-1065 (2010).

16. Kendler, K. S. \& Myers, J. The boundaries of the internalizing and externalizing genetic spectra in men and women. Psychological Med. 44, 647-655 (2013).

17. Krueger, R. F. et al. Etiological connections among substance dependence, antisocial behavior and personality: modeling the externalizing spectrum. $J$. Abnorm. Psychol. 111, 411-424 (2002).

18. Kendler, K. S., Prescott, C. A., Myers, J. \& Neale, M. C. The structure of genetic and environmental risk factors for common psychiatric and substance use disorders in men and women. Arch. Gen. Psychiatry 60, 929-937 (2003).

19. Bucholz, K. K. et al. A new, semi-structured psychiatric interview for use in genetic linkage studies: a report on the reliability of the SSAGA. J. Stud. Alcohol 55, 149-158 (1994).

20. Kaprio, J. The Finnish Twin Cohort Study: an update. Twin Res. Hum. Genet 16, 157-162 (2013).

21. Bucholz, K. K. et al. Comparison of parent, peer, psychiatric, and cannabis use influences across stages of offspring alcohol involvement: evidence from the COGA prospective study. Alcohol. Clin. Exp. Res. 41, 359-368 (2017).

22. Edenberg, H. J. The collaborative study on the genetics of alcoholism: an update. Alcohol Res. Health. 26, 214-218 (2002).
23. Begleiter, $\mathrm{H}$. et al. The collaborative study on the genetics of alcoholism. Alcohol Health Res. World 19, 228-228 (1995).

24. Martin, A. R. et al. Human Demographic History Impacts Genetic Risk Prediction across Diverse Populations. Am. J. Hum. Genet 100, 635-649 (2017).

25. American Psychiatric Association. Diagnostic and statistical manual of mental disorders (DSM-5 ${ }^{\circledR}$ ) (American Psychiatric Pub 2013).

26. Ge, T., Chen, C.-Y., Ni, Y., Feng, Y.-C. A. \& Smoller, J. W. Polygenic prediction via Bayesian regression and continuous shrinkage priors. Nat. Commun. 10, 1776 (2019).

27. Nakagawa, S., Schielzeth, H. \& O'Hara, R. B. A general and simple method for obtaining R-squared from generalized linear mixed-effects models. Methods Ecol. Evolution 4, 133-142 (2013).

28. Wald, N. J. \& Old, R. The illusion of polygenic disease risk prediction. Genet. Med. 21, 1705-1707 (2019).

29. Hanley, J. A. \& McNeil, B. J. The meaning and use of the area under a receiver operating characteristic (ROC) curve. Radiology 143, 29-36 (1982).

30. Benjamini, Y. \& Hochberg, Y. Controlling the false discovery rate: a practical and powerful approach to multiple testing. J. R. Stat. Soc. Ser. B (Methodol.) 57, 289-300 (1995).

31. Sanchez-Roige, S., Palmer, A. A. \& Clarke, T.-K. et al. Recent efforts to dissect the genetic basis of alcohol use and abuse. Biol. Psychiatry $\mathbf{8 7}$, 609-618 (2019).

32. Barr, P. B. \& Dick, D. M. The genetics of externalizing problems. Curr. Top. Behav Neurosci. https://link.springer.com/chapter/10.1007\%2F7854_2019_120 (2019)

33. Elliott, J. et al. Predictive accuracy of a polygenic risk score-enhanced prediction model vs a clinical risk score for coronary artery disease. JAMA $\mathbf{3 2 3}$, 636-645 (2020).

34. Abraham, G. et al. Genomic risk score offers predictive performance comparable to clinical risk factors for ischaemic stroke. Nat. Commun. 10, 5819 (2019).

35. Dudbridge, F. Power and Predictive Accuracy of Polygenic Risk Scores. PLoS Genet. 9, e1003348 (2013).

36. Grotzinger, A. D. et al. Genomic structural equation modelling provides insights into the multivariate genetic architecture of complex traits. Nat. Hum. Behav. 3, 513-525 (2019).

37. Using the Genetic Architecture of Externalizing Disorders and Behaviors to Aid in Gene Identification and Understanding Pathways of Risk. Proceedings of the Annual Meeting of the Behavior Genetics Association 2018. (European Neuropsychopharmacology).

38. Besterman, A. D., Moreno-De-Luca, D. \& Nurnberger, J. I. Jr. 21st-Century genetics in psychiatric residency training: how do we get there? JAMA Psychiatry 76, 231-232 (2019).

39. Nurnberger, J. I.Jr. et al. What should a psychiatrist know about genetics? review and recommendations from the residency education committee of the International Society of Psychiatric Genetics. J. Clin. Psychiatry 80, 17nr12046 (2018)

40. Martin, A. R. et al. Clinical use of current polygenic risk scores may exacerbate health disparities. Nat. Genet. 51, 584-591 (2019).

41. Kendler, K. S., Gardner, C. \& Dick, D. Predicting alcohol consumption in adolescence from alcohol-specific and general externalizing genetic risk factors, key environmental exposures and their interaction. Psychological Med. 41, 1507-1516 (2011).

42. Meyers, J. L. et al. Genetic influences on alcohol use behaviors have diverging developmental trajectories: a prospective study among male and female twins. Alcohol. Clin. Exp. Res. 38, 2869-2877 (2014).

43. Munafò, M. R., Tilling, K., Taylor, A. E., Evans, D. M. \& Davey Smith, G. Collider scope: when selection bias can substantially influence observed associations. Int. J. Epidemiol. 47, 226-235 (2017).

44. Barr, P. B. et al. Polygenic risk for alcohol misuse is moderated by romantic partnerships. Addiction 114, 1753-1762 (2019).

45. Cooke, M. E. et al. Gene-environment interaction effects of peer deviance, parental knowledge and stressful life events on adolescent alcohol use. Twin Res. Hum. Genet. 18, 507-517 (2015).

46. Dick, D. M. et al. The role of socioregional factors in moderating genetic influences on early adolescent behavior problems and alcohol use. Alcohol.: Clin. Exp. Res. 33, 1739-1748 (2009).

47. Dick, D. M. et al. Parental monitoring moderates the importance of genetic and environmental influences on adolescent smoking. J. Abnorm. Psychol. 116, 213-218 (2007). 